\title{
Role of the Gene Miniature in Drosophila Wing Maturation
}

\author{
Oleksii O. Bilousov, ${ }^{1,2,3}$ Iryna A. Kozeretska, ${ }^{1 \star}$ and Vladimir L. Katanaev ${ }^{2,3,4 *}$ \\ ${ }^{1}$ Department of General and Molecular Genetics, Taras Shevchenko National University of Kyiv, \\ Educational and Scientific Centre "Institute of Biology," Kyiv, Ukraine \\ ${ }^{2}$ Department of Pharmacology and Toxicology, University of Lausanne, Lausanne, Switzerland \\ ${ }^{3}$ Department of Biology, University of Konstanz, Universitätstrasse 10, Konstanz, Germany \\ ${ }^{4}$ Institute of Protein Research, Russian Academy of Sciences, Pushchino, Russia
}

Received 14 July 2011; Revised 20 January 2012; Accepted 21 January 2012

\begin{abstract}
Summary: Miniature is an extracellular zona pellucida domain-containing protein, required for flattening of pupal wing epithelia in Drosophila. Here, we show that Miniature also plays an important role in the post-eclosion wing maturation processes triggered by the neurohormone bursicon. Wing expansion and epithelial apoptosis are drastically delayed in miniature loss-of-function mutants, and sped up upon overexpression of the protein in wings. Miniature acts upstream from the heterotrimeric Gs protein transducing the bursicon signal in wing epithelia. We propose that Miniature interacts with bursicon and regulates its diffusion through or stability within the wing tissue. genesis 50:525-533, 2012. (c) 2012 Wiley Periodicals, Inc.
\end{abstract}

Key words: Drosophila; miniature; wing maturation; apoptosis; epithelial-to-mesenchymal transition; bursicon

The wing of a newly emerged Drosophila fruit fly is a soft folded structure. Soon after fly eclosion from the pupal case, release of the neurohormone bursicon from the brain and its delivery to the wing by hemolymph trigger a number of processes in wing epithelial cells, collectively referred to as wing maturation (Honegger et al., 2008). It includes epithelial-to-mesenchymal transition (EMT) and apoptosis of wing epithelia (Kimura et al., 2004; Natzle et al., 2008), accompanied by pumping of the hemolymph in between the dorsal and ventral wing layers and out to remove the cell debris (Togel et al., 2008). These processes result in unfolding and expansion of the wings. Additionally, adhesion of the two cuticle sheets, their sclerotization, and melanization take place, finally producing the flat firm flight organ of the adult insect (Johnson and Milner, 1987).
Binding of bursicon to the $G$ protein-coupled receptor Rickets on wing epithelia is considered the cause of these cellular events (Baker and Truman, 2002). Rickets activates the heterotrimeric Gs protein (Kimura et al., 2004; Luo et al., 2005), producing GTP-charged G $\alpha$ s

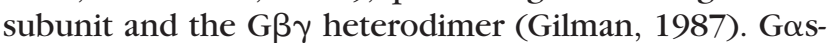
GTP activates the cAMP-PKA pathway responsible for induction of apoptosis (Kimura et al., 2004), while the G $\beta \gamma$ part appears to regulate the signaling branch controlling EMT and wing expansion (Katanayeva et al., 2010). Additionally, Tissue Inhibitor of metalloproteinases, integrins, and $\beta$-catenin has been implicated in Drosopbila wing maturation (Brower and Jaffe, 1989; Kiger et al., 2007).

The $\mathrm{X}$ chromosome-localized miniature $(m)$ gene is required for the late stages of pupal wing development (Roch et al., 2003). Its role is conserved among Drosopbila species, as mutations in this gene, both in $D$. melanogaster and $D$. virilis, result in about 1.5 -fold reduced wing surface without decrease in cell number (Dobzhansky, 1929; Kozeretska et al., 2004). Ultrastructural investigation revealed a defect in flattening of the

${ }^{*}$ Correspondence to: Iryna A. Kozeretska, Department of General and Molecular Genetics, Taras Shevchenko National University of Kyiv, Educational and Scientific Centre "Institute of Biology," Kyiv, Ukraine.

E-mail: iryna.kozeretska@gmail.com (or)

Vladimir L. Katanaev, Department of Pharmacology and Toxicology, University of Lausanne, Rue du Bugnon 27, CH-1005 Lausanne, Switzerland.

E-mail: Vladimir.Katanaev@unil.ch

Contract grant sponsors: Deutsche Forschungsgemeinschaft; University of Konstanz; Partnership Program of the University of Konstanz with Taras Shevchenko National University of Kyiv.

Published online 30 January 2012 in

Wiley Online Library (wileyonlinelibrary.com).

DOI: $10.1002 /$ dvg. 22016 

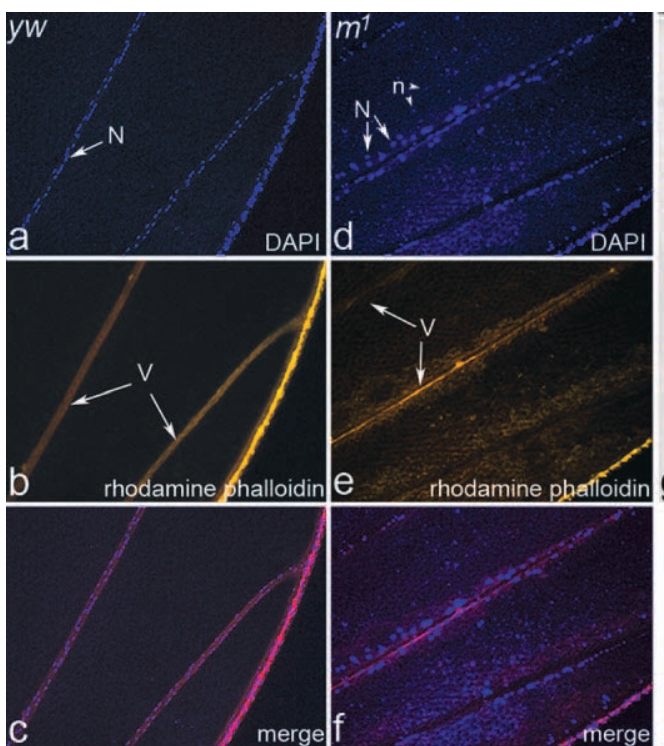

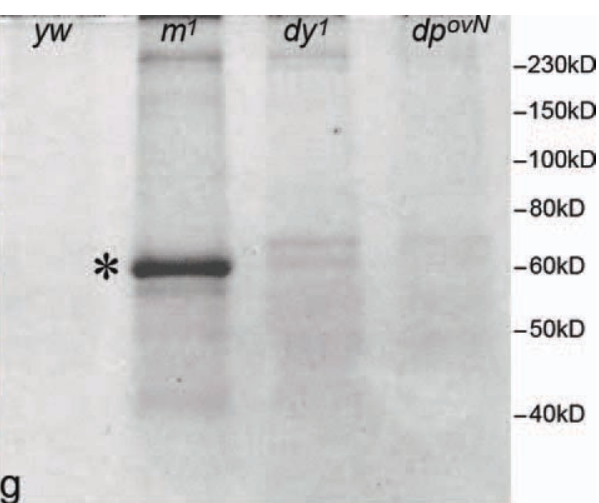

g

FIG. 1. Apoptosis and hemolymph removal are incomplete in miniature mutant wings. Wings of aged ( $>1 \mathrm{~d}$ post-eclosion) $y w$ (a-c) and $m^{1}$ (d-f) flies were stained with DAPI $(a, d)$ and rhodamine phalloidin $(b, e)$. While control wings show surviving cells only along the veins and wing margin $(\mathrm{a}-\mathrm{c})$, adult $\mathrm{m}^{1}$ wings contain many remaining cells scattered across the wing blade (d-f). $\mathrm{V}$ : veins; $\mathrm{N}$ : nuclei; $\mathrm{n}$ : fragmented nuclei; g: analysis of the protein composition of $y w, m^{1}, d y^{1}$, and $d p^{o v N}$ wings displays a dramatic increase in protein content in miniature mutant wings. The major band of the $m^{1}$ wing extracts identified as apolipophorin-2 is indicated with a star.

initially columnar wing epithelial cells, normally happening between 32 and $60 \mathrm{~h}$ after puparium formation, to be responsible for the overall reduced wing appearance in miniature mutants (Roch et al., 2003). Additionally, incomplete adherence of the wing epithelial sheets and wing hair orientation defects have been described in miniature mutants (Newby et al., 1991).

The protein encoded by the miniature gene has a signal peptide, a large extracellular portion with the zona pellucida (ZP)-domain and multiple glycosylation sites, a single transmembrane region, and a short intracellular tail. Since the transmembrane region is preceded by a furin-type endopeptidase cleavage site, the processed Miniature is assumed an extracellular protein (Jovine et al., 2005; Roch et al., 2003). The ZP-domain is an extracellular matrix (ECM) polymerization module and delineates a family of extracellular proteins with evolutionary conserved ECM organization functions (Jovine et al., 2005). ECM proteins control numerous tissue activities, including regulation of diffusion of secreted signaling molecules such as morphogens and hormones (Hynes, 2009). Since miniature is strongly expressed in wing epithelia and affects the properties of the wing ECM (Ren et al., 2005; Roch et al., 2003), we questioned whether the wing maturation events triggered by bursicon might be regulated by Miniature.

Mature adult wings of Drosopbila are mostly dead structures, with only vein and sensory cells remaining live (Fig. 1a-c). Cell debris could be seen in adult wings double mutant for miniature and its closely related ZP- domain protein encoding gene dusky (Roch et al., 2003), hinting at potential incompletion of wing maturation in these mutants. Indeed, our analysis of the mutant line $m^{1}$, presumed null allele of miniature (Roch et al., 2003), revealed that a significant amount of nuclear (DAPI) and cytoskeletal (rhodamine phalloidin) staining persisted in old ( $>1$ day) $m^{1}$ wings (Fig. 1d-f). DAPI staining of adult wings identified both intact and apparently fragmented cell nuclei (Fig. 1d). Thus, wing cell death appears incomplete in $m^{1}$ animals.

To further characterize old ( $>1$ day) $m^{1}$ wings, we performed SDS-PAGE of their protein content, along with that of control wings. Despite the equal quantity of the source material and identical extraction conditions, much more protein could be extracted from $m^{1}$ wings (Fig. 1g), in agreement with the persistence of cells and their debris in these wings. We performed similar protein extraction from wings of $d y^{1}$ and $d p^{o v N}$ mutants: loss-of-function alleles of dusky, which is involved jointly with miniature in flattening of the pupal wing epithelia (DiBartolomeis et al., 2002; Roch et al., 2003; Waddington, 1940), and dumpy, encoding another ZP-domain protein (Wilkin et al., 2000), respectively. $d y^{1}$ wings contained much less protein than $m^{1}$, while protein content of $d p^{o v N}$ wings was essentially indistinguishable from wild-type.

By peptide mass-spectrometry, we identified the major protein band of the $m^{1}$ wing extracts, migrating at $61 \mathrm{kDa}$ (Fig. 1g), as apolipophorin-2. This protein is secreted into the hemolymph by the fat bodies and it is one of the major hemolymph clot proteins (Karlsson 

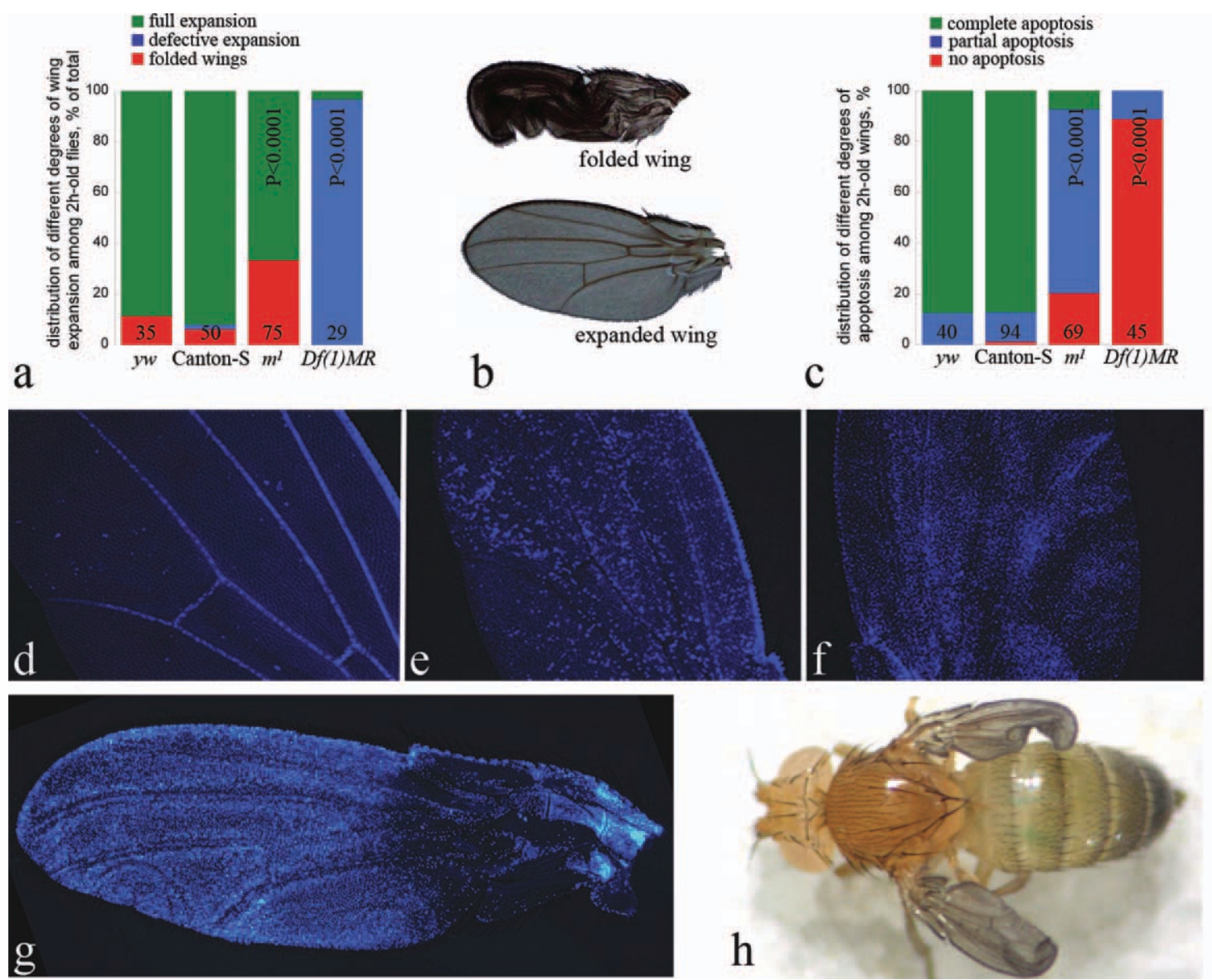

FIG. 2. Wing expansion and apoptosis are dramatically delayed in miniature mutants. (a) Quantification of distribution of different degrees of wing expansion (full expansion, defective expansion, and folded wings) among $2 \mathrm{~h}$ post-eclosion control ( $y w$ and Canton-S) and miniature mutant $\left[^{1}\right.$ and $\left.D f(1) M R\right]$ flies. Numbers of flies analyzed is given at the basis of the bars. $P$-value of differences of the mutant genotypes from both control genotypes is from the Pearson's chi-squared test. (b) Examples of folded and expanded wings of the yw genotype. (c) Quantification of distribution of different degrees of apoptosis (complete, partial, or no apoptosis) among $2 \mathrm{~h}$ post-eclosion control (yw and Canton-S) and miniature mutant $\left[\mathrm{m}^{1}\right.$ and $\left.D f(1) M R\right]$ wings. Numbers of wings analyzed is given at the basis of the bars. $P$-value of differences of the mutant genotypes from both control genotypes is from the Pearson's chi-squared test. (d-f): Examples of $2 \mathrm{~h}$ post-eclosion wings having complete apoptosis $(y w, d)$, partial apoptosis $\left(m^{1}\right.$, e), and no apoptosis $\left(m^{1}, \mathrm{f}\right)$. (g and $\left.\mathbf{h}\right) D f(1) M R$ mutant animals show strongly enhanced phenotype of delayed apoptosis and at the same time incompletion in wing expansion and flattening. (g) Most $2 \mathrm{~h}$ post-eclosion $D f(1) M R$ flies have no apoptosis in wings. (h) $D f(1) M R$ flies display defects in flattening their severely reduced wing. Wings in $\mathrm{d}-\mathrm{g}$ are shown in scale.

et al., 2004; Kutty et al., 1996; Scherfer et al., 2004). Its presence in wings of the adult mutant flies implies that hemolymph is not properly flushed off the $m^{1}$ wings and clots there.

To analyze whether wing maturation processes are indeed delayed in miniature mutants, we analyzed $m^{1}$ and $m^{+}$wings at $2 \mathrm{~h}$ post-eclosion, when EMT, apoptosis, and wing expansion are normally mostly completed (Johnson and Milner, 1987; Kiger et al., 2007; Kimura et al., 2004). Surprisingly, we found that $33 \%$ of $m^{1}$ flies did not expand their wings by that time, as compared with only about $10 \%$ of the control animals (Fig. 2a,b). By the Pearson's chi-squared test, this difference is highly statistically significant $(P<0.0001)$. As wing expansion strongly correlates with EMT (Katanayeva et al., 2010; Kiger et al., 2007), we hypothesized that EMT is delayed in $m^{1}$ flies. Relocalization of arm-GFP from cell boundaries to cytoplasm is typically used to monitor EMT in Drosophila wings (Kiger et al., 2007; Natzle et al., 2008). Unfortunately, strong cuticle autofluorescence of $m^{1}$ wings (much stronger than in $m^{+}$ wings) prevented us from the direct investigation of EMT in this and other (see below) miniature mutant genetic backgrounds.

To monitor apoptosis, we performed DAPI staining of $2 \mathrm{~h}$ post-eclosion $m^{1}$ and $m^{+}$wings and found a striking delay in apoptosis in $m^{1}$ flies (Fig. 2c-f). While about $90 \%$ of control wings have undergone complete apoptosis during the $2 \mathrm{~h}$ post-eclosion (Fig. 2c,d), intensive DAPI staining in $m^{1}$ wings revealed that the vast major- 
ity of $m^{1}$ animals had at best only partially completed cell death of their wing epithelia by that time (Fig. $2 \mathrm{c}, \mathrm{e}, \mathrm{f})$. We separately scored $2 \mathrm{~h}$-old wings as having no apoptosis (most wing blade area [ $>80 \%$ ] still filled with nuclei), partial apoptosis (roughly $20-80 \%$ of persisting nuclei), and complete apoptosis (more than $80 \%$ of area lacking nuclei). When scored in this manner, $m^{1}$ wings grouped as $20 / 73 / 7 \%$ (no/partial/complete apoptosis), as opposed to the control wings where $87 \%$ had complete apoptosis (Fig. 2c). The difference between the two genotypes is highly statistically significant $(P<$ 0.0001 ) by the Pearson's chi-squared test.

The $m^{1}$ mutation we used so far is a null or strong hypomorph allele of miniature (Roch et al., 2003). To exclude the possibility that the phenotypes we observed are due to a second site mutation on the $m^{1}$. carrying chromosome, we studied other mutations: $m^{D}$ that is a dominant mutant but allelic to both miniature and dusky and $m^{259-4}$, which is a small lethal deficiency not covering dusky (Slatis and Willermet, 1954), and two deficiencies removing both miniature and dusky genes: small viable deficiency $D f(1) M R$ (Roch et al., 2003) and a bigger lethal deficiency $D f(1) B S C 541$. Analysis of these alleles and their transheterozygous combinations provides interesting insights (Table 1; Fig. 2).

First, we clearly establish that the delayed apoptosis phenotype of $m^{1}$ is specific to the miniature mutation, as all other mutant combinations (hemizygous males, homozygous females where viable mutations were used, as well as transheterozygous females) also depict a drastic delay in apoptosis (Table 1; Fig. 2).

Second, we find that in some mutant backgrounds, specifically $m^{D} / Y, \quad m^{D} / m^{D}, \quad D f(1) M R / Y, \quad D f(1) M R /$ $D f(1) M R$, and $D f(1) M R / D f(1) B S C 541$, the phenotype of delayed apoptosis is strongly enhanced, such that, for example, in $D f(1) M R$ flies almost $90 \%$ of $2 \mathrm{~h}$ post-eclosion wings had no apoptosis as opposed to the 20/73/ $7 \%$ (no/partial/complete apoptosis) distribution in $m^{1}$ wings (Fig. 2c,g; Table 1). It is noteworthy that these enhanced phenotypes are produced by mutation combinations affecting both miniature and dusky genes. It thus appears that Dusky can partially rescue the miniature phenotype of delayed apoptosis, similarly to as it does in case of regulation of wing size and late-pupal flattening of wing epithelia, even though dusky mutations by themselves produce only weak defects in the wing size (Roch et al., 2003; Slatis and Willermet, 1954). In this regard, it is worth pointing out that $d y^{1}$ wings do maintain certain-even though much more modest than $m^{1}$-amounts of protein in their wings (see Fig. 1g).

Third, while the $m^{D}$ mutation is clearly dominant in the sense of affecting wing size as reported previously (Slatis and Willermet, 1954), it shows no dominance in the delay of apoptosis. Indeed, $m^{D} /+2 \mathrm{~h}$ post-eclosion wings show as complete apoptosis as the Canton-S and

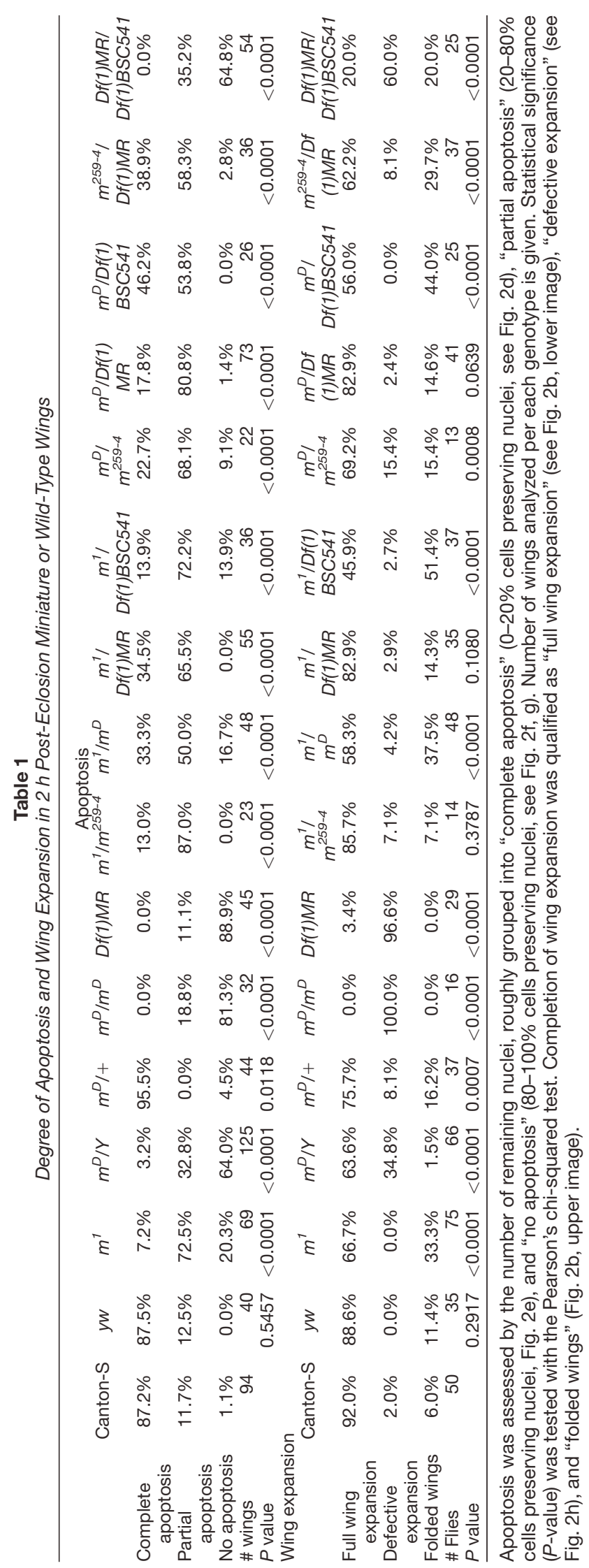


$y w$ controls, as opposed to severely delayed apoptosis in $m^{D} / Y$ and $m^{D} / m^{D}$ flies (Table 1). This data may suggest that the mechanism of action of Miniature in the regulation of wing maturation is molecularly distinct from that in the earlier epithelial flattening program.

Fourth, not all miniature mutant combinations display the clear wing unfolding defect frequently seen in $m^{1}$ wings (Fig. 2a,b). Of the mutants studied, the following show strong penetrance (30-50\% of flies) of this phenotype: $m^{259-4} / D f(1) M R, m^{D} / D f(1) B S C 541, m^{1} /$ $D f(1) B S C 541$, and $m^{1} / m^{D}$ (Table 1). In contrast, other mutants revealed $0-20 \%$ of $2 \mathrm{~h}$ post-eclosion flies with such defect. These frequencies are rather typical for the wild-type control flies ( $y w$ and Canton-S, Table 1). However, we note that some miniature mutants, not showing the clear wing unfolding defect as that of Figure $2 b$, still revealed incompletion in wing expansion and flattening, such as shown on Figure $2 \mathrm{~h}$ for a $D f(1) M R / Y$ fly. When such incomplete wing unfolding is taken into account, most miniature mutants analyzed corroborate with $m^{1}$ in terms of defective wing expansion (Table 1; Fig. 2a). Moreover, these phenotypes, as was found above for the delayed apoptosis, become more penetrant in the mutant backgrounds affecting both miniature and dusky genes (e.g., $m^{D} / m^{D}, D f(1) M R /$ $D f(1) M R$, and $D f(1) M R / D f(1) B S C 541)$ where it reaches $80-100 \%$ of flies (Table 1 ; Fig. 2a). We propose, based on previous findings (Katanayeva et al., 2010; Kiger et al., 2007), that these wing expansion defects in miniature mutants reflect delayed EMT. Differences between the "choice" of the phenotype [such as folded wings in $m^{1}$ vs. incomplete flattening in $m^{D}$ or $D f(1) M R]$ may depend on other factors, for example, the stronger reduction in the wing size in the $m^{D}$ and $D f(1) M R$ flies [see Fig. 2 and (Slatis and Willermet, 1954)]. Other, miniature-independent genetic factors may contribute to the penetrance of this phenotype (see below). In any respect, we stress that without direct EMT analysis the molecular significance of these wing expansion defects must be inferred with caution.

The above observations clearly demonstrate that Miniature is required for proper wing maturation. We next performed an epistasis experiment to roughly place Miniature in the hierarchy of the bursicon-initiated signaling steps leading to post-eclosion wing maturation. Expression of the GTPase-deficient (constitutively active) $\alpha$-subunit of the heterotrimeric Gs protein (G $\alpha s[G T P])$ in wing tissue leads to precocious apoptosis; as a result, the dorsal and ventral wing sheets fail to adhere and hemolymph accumulates inside the wing producing blistered wings (Katanayeva et al., 2010; Kimura et al., 2004; Wolfgang et al., 1996). Activation of the Gos-cAMP-PKA cascade is able to trigger apoptosis even when the neurohormone access to the wing epithelia is prevented by neck-ligation of pharate adults immediately after eclosion (Kimura et al., 2004).
The dominant wing blistering phenotype of Gos[GTP]-expressing flies can be used to assess whether Miniature acts upstream of Gs (that is, at the level of bursicon or Rickets) or downstream of Gs (at the level of cAMP-PKA or cell death machinery) in the regulation of apoptosis. If Miniature acted downstream from Gs, Gas[GTP] would be unable to induce wing blistering in the $m^{1}$ genetic background (or its ability to induce blistering would be reduced). In contrast, if Miniature acted upstream from Gs, its removal would not affect the phenotype of G $\alpha s[\mathrm{GTP}]$ expression.

We used OK10-Gal4, a wing-specific line driving expression weakly throughout the prospective wing blade at third larval stages and strongly throughout the wing blade at late pupal stages (Wolfgang et al., 1996). As reported previously (Katanayeva et al., 2010; Wolfgang et al., 1996), expression of Gos[GTP] by OK10Gal4 leads to formation of blisters in $100 \%$ of flies (Fig. $3 a)$. Importantly, the same was found in $\mathrm{m}^{1}$; OK10Gal4, UAS-G $\alpha s[G T P]$ flies (Fig. 3b). Thus, we conclude that Miniature acts in the regulation of the wing maturation processes upstream from the heterotrimeric Gs protein.

Miniature is expressed by several cuticle-secreting tissues (Roch et al., 2003) but not by most of other tissues (Chintapalli et al., 2007; Fernandes et al., 2010); of note, it is not detected in the nervous system where bursicon is produced (Honegger et al., 2008). To prove further that the action of Miniature in post-eclosion wing maturation is wing-autonomous, we overexpressed miniature in wings using the OK10-Gal4 driver. Since apoptosis is already mostly complete by 2 $\mathrm{h}$ post-eclosion in wild-type flies (Table 1), we compared flies $1 \mathrm{~h}$ post-eclosion without and with overexpression of miniature. Remarkably, we find that OK10-Gal4; UAS-miniature flies have markedly advanced apoptosis by $1 \mathrm{~h}$ post-eclosion (Fig. 3c,d, Table 2). At the same time, no blisters were ever seen in these flies, as opposed to those expressing Gos[GTP] in the same manner. Thus, it might be speculated that overexpression of miniature can speed-up bursiconinduced apoptosis, but does not induce precocious (bursicon-independent) apoptosis which is stimulated by activation of signaling at the level of G $\alpha$ s[GTP] or below (Katanayeva et al., 2010; Kimura et al., 2004; Wolfgang et al., 1996).

We also tested other Gal4 drivers to overexpress miniature, as well as different UAS-miniature insertion lines. Of the driver lines tested, 71B-Gal4 drives expression in the whole wing pouch excluding the margin (Capdevila and Guerrero, 1994), while engrailed-Gal4 (en-Gal4) and bedgebog-Gal4 drive expression only in the posterior half of the wing. Previ-

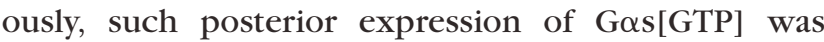
found effective in inducing precocious cell death (Kimura et al., 2004). We find that these Gal4 driver 


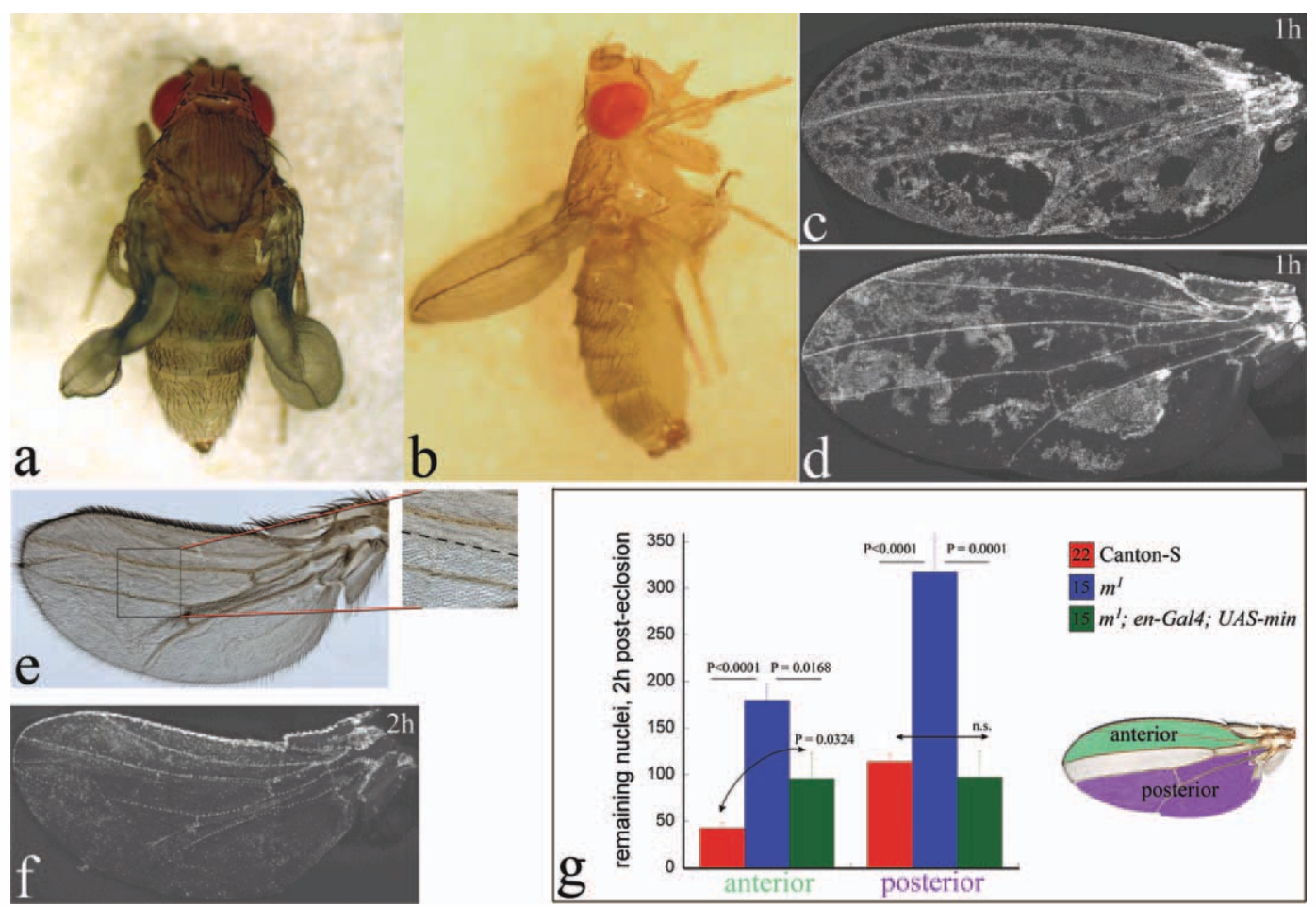

FIG. 3. Wing-expressed miniature functions upstream from the heterotrimeric Gs protein to regulate wing maturation in a nonautonomous

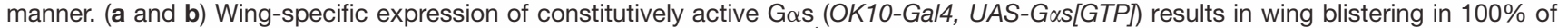
pharate adults either wild-type (a) or mutant (b) for the miniature gene $\left(\mathrm{m}^{1}\right)$. (c and d) $1 \mathrm{~h}$ post-eclosion wild-type (Canton-S) wings have only begun their apoptosis (c), as opposed to OK10-Gal4; UAS-miniature flies which have markedly advanced apoptosis (d). (e and f) en-Gal4; UAS-miniature rescues miniature loss-of-function $(D f(1) M R)$. While the rescue in overall wing size and wing cell cross-section dimension is strictly compartment specific (e, compartment border is shown with the dashed line on insert), apoptosis delay is efficiently rescued in both posterior and anterior compartments (f). (g) Quantification of surviving nuclei in $2 \mathrm{~h}$ post-eclosion wings of wild-type (Canton-S), mutant $\left(\mathrm{m}^{1}\right)$, and posteriorly rescued flies $\left(\mathrm{m}^{1}\right.$; en-Gal4; UAS-miniature) in the anterior and posterior compartments of the wings. Rescue in the posterior compartment is complete; rescue in the anterior compartment is partial but significant, revealing the nonautonomous action of Miniature in the wing. Insert shows the areas where quantification was performed. Numbers next to the genotype descriptions show the number of wings analyzed. P-values from the Student $t$-test show high statistical significance of differences between the genotypes; "n.s.": nonsignificant $(P>0.05)$.

Table 2

Degree of Apoptosis in $1 \mathrm{~h}$ Post-Eclosion UAS-Miniature or Wild-Type Wings

\begin{tabular}{|c|c|c|c|c|c|c|}
\hline & Canton-S & $71 B-G a / 4$ & UAS-min; OK10-Gal4 & UAS-min; 71B-Gal4 & en-Gal4; UAS-min & UAS-min; hh-Gal4 \\
\hline Complete apoptosis & $4.9 \%$ & $11.5 \%$ & $17.1 \%$ & $24.4 \%$ & $32.0 \%$ & $35.7 \%$ \\
\hline Partial apoptosis & $21.3 \%$ & $30.8 \%$ & $65.9 \%$ & $44.4 \%$ & $32.0 \%$ & $31.0 \%$ \\
\hline No apoptosis & $73.8 \%$ & $57.7 \%$ & $17.1 \%$ & $31.1 \%$ & $36.0 \%$ & $33.3 \%$ \\
\hline \# Wings & 61 & 26 & 41 & 45 & 25 & 42 \\
\hline$P$ value & & 0.1153 & $<0.0001$ & $<0.0001$ & $<0.0001$ & $<0.0001$ \\
\hline
\end{tabular}

Analysis was performed as in Table 1.

lines were all effective in speeding up apoptosis (Table 2). Interestingly, UAS-miniature; $71 B$-Gal4 flies also display more advanced wing expansion as compared to the parental $71 B$-Gal4 driver line in which wing expansion is retarded compared with other Gal4 and wild-type lines: $42.9 \%$ of $1 \mathrm{~h}$ post-eclosion $71 \mathrm{~B}$-Gal4 flies have folded wings as opposed to $28.1 \%$ in UASminiature; $71 B$-Gal4 ( $n=28-32$ flies); this difference is highly statistically significant by the Pearson's chisquared test $(P<0.0001)$. Other miniature overex- pression combinations were not different in their wing expansion from the parental Gal4 lines.

Finally, we used one of these overexpression combinations (en-Gal4; UAS-miniature) to rescue miniature loss-of-function phenotypes $\left[D f(1) M R\right.$ and $\left.m^{1}\right]$. Such posterior expression could clearly provide compartment-autonomous rescue of the cell size, resulting in an increase in the posterior wing area (Fig. 3e,f, and insert on Fig. 3e). We next tested whether the delay in wing maturation processes of $m^{1}$ and $D f(1) M R$ flies would 
Table 3

Degree of Apoptosis and Wing Expansion in 2 h Post-Eclosion Miniature-Rescue Wings

\begin{tabular}{|c|c|c|c|c|c|c|}
\hline \multicolumn{7}{|c|}{ Apoptosis } \\
\hline & Canton-S & en-Gal4 & $m^{1}$ & $m^{1}$; en-Gal4; UAS-min & $D f(1) M R$ & Df(1)MR; en-Gal4; UAS-min \\
\hline Complete apoptosis & $87.2 \%$ & $95.8 \%$ & $7.2 \%$ & $87.0 \%$ & $0.0 \%$ & $21.4 \%$ \\
\hline Partial apoptosis & $11.7 \%$ & $4.2 \%$ & $72.5 \%$ & $8.7 \%$ & $11.1 \%$ & $57.1 \%$ \\
\hline No. apoptosis & $1.1 \%$ & $0.0 \%$ & $20.3 \%$ & $4.3 \%$ & $88.9 \%$ & $21.4 \%$ \\
\hline \# Wings & 94 & 71 & 69 & 23 & 45 & 14 \\
\hline$P$ value & & & & $<0.0001$ & & $<0.0001$ \\
\hline \multicolumn{7}{|c|}{ Wing expansion } \\
\hline & Canton-S & en-Gal4 & $m^{1}$ & $m^{1}$; en-Gal4; UAS-min & $D f(1) M R$ & Df(1)MR; en-Gal4; UAS-min \\
\hline Full wing expansion & $92.0 \%$ & $100.0 \%$ & $66.7 \%$ & $100.0 \%$ & $3.4 \%$ & $87.5 \%$ \\
\hline Defective expansion & $2.0 \%$ & $0.0 \%$ & $0.0 \%$ & $0.0 \%$ & $96.6 \%$ & $0.0 \%$ \\
\hline Folded wings & $6.0 \%$ & $0.0 \%$ & $33.3 \%$ & $0.0 \%$ & $0.0 \%$ & $12.5 \%$ \\
\hline \# Flies & 50 & 39 & 75 & 12 & 29 & 8 \\
\hline$P$ value & & & & $<0.0001$ & & $<0.0001$ \\
\hline
\end{tabular}

Analysis was performed as in Table 1.

be rescued by such expression. And indeed, both apoptosis and wing expansion defects of $m^{1}$ and $D f(1) M R$ were rescued to a large extent (Table 3; compare Fig. $3 \mathrm{f}$ with Fig. 2g). We were puzzled by the efficient rescue of the apoptosis phenotype in both the posterior and anterior compartments (Fig. 3f) which drastically differed from the rescue of the cell size, which was strictly compartment-autonomous. Thus, we analyzed more carefully the amounts of surviving nuclei in $2 \mathrm{~h}$ posteclosion wings of wild-type, mutant, and posteriorly rescued flies in the anterior and posterior compartments. To avoid confusion at the border of the two compartments, we counted remaining nuclei in the part of the anterior compartment between wing margin and vein 3 , while the posterior analysis was restricted to the area between vein 4 and the posterior margin (as schematized in insert of Fig. 3g). In this manner, more nuclei can be counted in the posterior region of $2 \mathrm{~h}$ post-eclosion Canton-S flies than in the anterior area (Fig. $3 \mathrm{~g}$ ). $\mathrm{m}^{1}$ mutants, as described above, show markedly increased numbers of nuclei in both areas. en-Gal4; UAS-miniature is found to completely rescue wing apoptosis to the wild-type levels in the posterior domain of the $m^{1}$ wings (Fig. 3g). Remarkably, we find that the anterior region is also significantly rescued, although to the levels clearly below those of the posterior area. Altogether, these findings reveal a nonautonomous ability of Miniature to rescue wing maturation processes in $m^{1}$ and $D f(1) M R$ flies.

Components of the ECM can bind secreted molecules-hormones and morphogens-and regulate their diffusion, stability, and activity (Hynes, 2009). For example, type IV collagen directly binds Dpp, a Drosophila member of the TGF $\beta$ family of secreted growth factors, to regulate its diffusion through developing tissues, contributing to Dpp signaling (Wang et al., 2008). Similarly, ECM protein periostin expressed by stromal cells binds and accumulates Wnt ligands, which are critical for colonization of secondary tissues by metastatic cancer cells
(Malanchi et al., 2012). We have found that a ZP-domain extracellular protein Miniature (and potentially its close homolog Dusky) is required for the post-eclosion maturation processes in Drosopbila wings, which are initiated by the neurohormone bursicon. Miniature acts within the wing tissue, regulating the epithelial response to bursicon. Removal of Miniature markedly slows down apoptosis and potentially EMT in wing epithelia. Miniature acts upstream from the bursicon-transducing heterotrimeric Gs protein in these events and, unlike activated $G \alpha s$, is unable to induce precocious apoptosis, although it does accelerate it upon overexpression. Finally, re-expression of Miniature rescues miniature mutant conditions to the full extent cell-autonomously, but also to a certain extent in a nonautonomous manner within the wing. Cumulatively, these findings speak for a function of Miniature in the regulation of diffusion/stability of bursicon through the pharate wing tissue, similarly to the examples of ECM-growth factor interactions described above. We propose that Miniature traps bursicon in the ECM, increasing its concentration or longevity of its presence for higher activation of the wing maturation processes. When re-expressed posteriorly in miniature mutants, the protein creates a posterior "sink" of the neurohormone, which can then diffuse anteriorly to trigger signaling also in this compartment.

Bursicon belongs to a large cysteine knot protein family of secreted factors and is mostly related to the vertebrate BMP (bone morphogenetic protein) antagonists such as Gremlin and Cerberus (Honegger et al., 2008; Luo et al., 2005; Vitt et al., 2001), which play important roles in execution of BMP-dependent developmental programs (Walsh et al., 2010). Similarly, the extracellular ZP-domain proteins are well conserved in animal evolution (Jovine et al., 2005). We propose that Miniature directly regulates bursicon diffusion and activity in flies, and that their homologs may similarly interact in vertebrates. 


\section{MATERIALS AND METHODS}

\section{Fly Stocks and Crosses}

The following Drosopbila lines were used: OK10Gal4 and UAS-Gas[GTP] (Wolfgang et al., 1996), bhGal4 (Tanimoto et al., 2000), UAS-miniature [insertion lines on chromosomes 2 and 3, (Fernandes et al., 2010)], Df(1)MR (Roch et al., 2003). Canton-S, $y w, d y^{1}$, $d p^{o v N}, m^{1}, m^{D}, m^{259-4}, D f(1) B S C 541,71 B-G a l 4$, enGal4, and arm-GFP were from the Bloomington Drosopbila Stock Center. All crosses were performed at $25^{\circ} \mathrm{C}$.

\section{Protein Assays}

Protein extraction was carried out from 50 wings of adult ( $>1$ day-old) flies. Wings were frozen in liquid nitrogen and homogenized in SDS-PAGE buffer by 1 h-intensive vortexing with glass beads, followed by $0.5 \mathrm{~h}$ incubation at $55^{\circ} \mathrm{C}$. Before applying to $10 \%$ SDS-PAGE, the samples were additionally boiled for $10 \mathrm{~min}$. Four independent preparations with identical results were conducted. In-gel trypsin digestion and mass-spectrometry was performed by the Proteomics Facility of the University of Konstanz (Germany).

\section{Microscopy}

Whole flies were photographed through a Carl Zeiss Stemi 2000 binocular using the Canon PowerShot G10 camera. Adult wings were mounted as described (Katanaev et al., 2005). For fluorescence labeling, wings were fixed in 4\%-formaldehyde/0.2\% Tween-20 in PBS (20 min RT), washed three times in $0.2 \%$ Tween-20/PBS at RT, dehydrated in chloroform $\left(1 \mathrm{~h} ; 17^{\circ} \mathrm{C}\right)$, dechitinated in heptane $\left(3 \mathrm{~h} ; 17^{\circ} \mathrm{C}\right)$, and washed again in $0.2 \%$ Tween-20/PBS ( $3 \mathrm{~h} ; 17^{\circ} \mathrm{C}$ ) before overnight staining with DAPI (1:10,000 dilution, Sigma) and $0.5 \mu \mathrm{M}$ rhodamine phalloidin (Invitrogen) at $4{ }^{\circ} \mathrm{C}$. Excess of the dies was removed with three washings prior to mounting the wings in Vectashield for fluorescence microscopy (AxioImager, Carl Zeiss). Analysis of the degree of apoptosis was qualitative (except for Fig. 3g), based on investigation of overall several hundreds of DAPI-stained wings, which could be easily grouped into those depicting "no apoptosis" (whole wing blade area filled with nuclei, with DAPI-negative cell outlines corresponding to no more than roughly $20 \%$ of the area), "complete apoptosis" (whole wing area devoid of nuclei, with DAPIpositive cells corresponding to no more than roughly $20 \%$ of the area), and the intermediate "partial apoptosis." Fully folded wings were excluded from the apoptosis analysis due to difficulty in visualization.

\section{ACKNOWLEDGMENTS}

The authors thank Michael Forte (Oregon Health and Science University), Fernando Roch (Université Paul
Sabatier, Toulouse), Serge Plaza (University of Toulouse), and the Bloomington Drosopbila Stock Center for fly lines, Alexey Koval for technical advice, and members of the Katanaev lab for critically reading the manuscript.

\section{LITERATURE CITED}

Baker JD, Truman JW. 2002. Mutations in the Drosophila glycoprotein hormone receptor, rickets, eliminate neuropeptide-induced tanning and selectively block a stereotyped behavioral program. J Exp Biol 205:2555-2565.

Brower DL, Jaffe SM. 1989. Requirement for integrins during Drosophila wing development. Nature 342:285-287.

Capdevila J, Guerrero I. 1994. Targeted expression of the signaling molecule decapentaplegic induces pattern duplications and growth alterations in Drosophila wings. EMBO J 13:4459-4468.

Chintapalli VR, Wang J, Dow JA. 2007. Using FlyAtlas to identify better Drosophila melanogaster models of human disease. Nat Genet 39:715-720.

DiBartolomeis SM, Akten B, Genova G, Roberts MA, Jackson FR. 2002. Molecular analysis of the Drosophila miniature-dusky ( $\mathrm{m}$-dy) gene complex: m-dy mRNAs encode transmembrane proteins with similarity to C. elegans cuticulin. Mol Genet Genomics 267:564-576.

Dobzhansky T. 1929. The influence of the quantity and quality of chromosomal material on the size of the cells in drosophila melanogaster. Dev Genes Evolution 115:363-379.

Fernandes I, Chanut-Delalande H, Ferrer P, Latapie Y, Waltzer L, Affolter M, Payre F, Plaza S. 2010. Zona pellucida domain proteins remodel the apical compartment for localized cell shape changes. Dev Cell 18:64-76.

Gilman AG. 1987. G proteins: Transducers of receptorgenerated signals. Annu Rev Biochem 56:615-649.

Honegger H-W, Dewey E, Ewer J. 2008. Bursicon, the tanning hormone of insects: Recent advances following the discovery of its molecular identity. J Comp Physiol A Neuroethol Sens Neural Behav Physiol 194:989-1005.

Hynes RO. 2009. The extracellular matrix: Not just pretty fibrils. Science 326:1216-1219.

Johnson SA, Milner MJ. 1987. The final stages of wing development in Drosophila melanogaster. Tissue Cell 19:505-513.

Jovine L, Darie CC, Litscher ES, Wassarman PM. 2005. Zona pellucida domain proteins. Annu Rev Biochem 74:83-114.

Karlsson C, Korayem AM, Scherfer C, Loseva O, Dushay MS, Theopold U. 2004. Proteomic analysis of the Drosophila larval hemolymph clot. J Biol Chem 279:52033-52041. 
Katanaev VL, Ponzielli R, Semeriva M, Tomlinson A. 2005. Trimeric G protein-dependent frizzled signaling in Drosophila. Cell 120:111-122.

Katanayeva N, Kopein D, Portmann R, Hess D, Katanaev VL. 2010. Competing activities of heterotrimeric G proteins in Drosophila wing maturation. PLoS ONE 5:e12331.

Kiger JA, Jr., Natzle JE, Kimbrell DA, Paddy MR, Kleinhesselink K, Green MM. 2007. Tissue remodeling during maturation of the Drosophila wing. Dev Biol 301:178-191.

Kimura K, Kodama A, Hayasaka Y, Ohta T. 2004. Activation of the cAMP/PKA signaling pathway is required for post-ecdysial cell death in wing epidermal cells of Drosophila melanogaster. Development 131:1597-1606.

Kozeretska IA, Gubenko IS, Gorb SN. 2004. New unusual miniature-like wing mutation in Drosophila virilis. J Morphol 261:270-275.

Kutty RK, Kutty G, Kambadur R, Duncan T, Koonin EV, Rodriguez IR, Odenwald WF, Wiggert B. 1996. Molecular characterization and developmental expression of a retinoid- and fatty acid-binding glycoprotein from Drosophila. A putative lipophorin. J Biol Chem 271:20641-20649.

Luo CW, Dewey EM, Sudo S, Ewer J, Hsu SY, Honegger HW, Hsueh AJ. 2005. Bursicon, the insect cuticlehardening hormone, is a heterodimeric cystine knot protein that activates $G$ protein-coupled receptor LGR2. Proc Natl Acad Sci U S A 102:2820-2825.

Malanchi I, Santamaria-Martinez A, Susanto E, Peng H, Lehr HA, Delaloye JF, Huelsken J. 2012. Interactions between cancer stem cells and their niche govern metastatic colonization. Nature 481:85-89.

Natzle JE, Kiger JA, Jr., Green MM. 2008. Bursicon signaling mutations separate the epithelial-mesenchymal transition from programmed cell death during Drosophila melanogaster wing maturation. Genetics 180:885-893.

Newby LM, White L, DiBartolomeis SM, Walker BJ, Dowse HB, Ringo JM, Khuda N, Jackson FR. 1991. Mutational analysis of the Drosophila miniaturedusky (m-dy) locus: Effects on cell size and circadian rhythms. Genetics 128:571-582.
Ren N, Zhu C, Lee H, Adler PN. 2005. Gene expression during Drosophila wing morphogenesis and differentiation. Genetics 171:625-638.

Roch F, Alonso CR, Akam M. 2003. Drosophila miniature and dusky encode ZP proteins required for cytoskeletal reorganisation during wing morphogenesis. J Cell Sci 116:1199-1207.

Scherfer C, Karlsson C, Loseva O, Bidla G, Goto A, Havemann J, Dushay MS, Theopold U. 2004. Isolation and characterization of hemolymph clotting factors in Drosophila melanogaster by a pullout method. Curr Biol 14:625-629.

Slatis HM, Willermet DA. 1954. The miniature complex in Drosophila melanogaster. Genetics 39:45-58.

Tanimoto H, Itoh S, ten Dijke P, Tabata T. 2000. Hedgehog creates a gradient of DPP activity in Drosophila wing imaginal discs. Mol Cell 5:59-71.

Togel M, Pass G, Paululat A. 2008. The Drosophila wing hearts originate from pericardial cells and are essential for wing maturation. Dev Biol 318:29-37.

Vitt UA, Hsu SY, Hsueh AJ. 2001. Evolution and classification of cystine knot-containing hormones and related extracellular signaling molecules. Mol Endocrinol 15:681-694.

Waddington $\mathrm{CH}$. 1940. The genetic control of wing development in Drosophila. J Gene 41:75-139.

Walsh DW, Godson C, Brazil DP, Martin F. 2010. Extracellular BMP-antagonist regulation in development and disease: Tied up in knots. Trends Cell Biol 20:244256.

Wang X, Harris RE, Bayston LJ, Ashe HL. 2008. Type IV collagens regulate BMP signalling in Drosophila. Nature 455:72-77.

Wilkin MB, Becker MN, Mulvey D, Phan I, Chao A, Cooper K, Chung HJ, Campbell ID, Baron M, MacIntyre R. 2000. Drosophila dumpy is a gigantic extracellular protein required to maintain tension at epidermal-cuticle attachment sites. Curr Biol 10:559567.

Wolfgang WJ, Roberts IJ, Quan F, O'Kane C, Forte M. 1996. Activation of protein kinase A-independent pathways by Gs alpha in Drosophila. Proc Natl Acad Sci U S A 93:14542-14547. 